\title{
Expression and Prognostic Value of Glucose Transporter 3 in Diffuse Large B Cell Lymphoma
}

\author{
Yongpeng Xu', Xinglu Zhou², Shuai Zhang ${ }^{3}$, Abiyasi Nanding ${ }^{4}$, Qijia Xuan ${ }^{5}$ \\ 'Department of Urology Surgery, The Fourth Affiliated Hospital Zhejiang University School of Medicine, Yiwu, Zhejiang Province, 322000, People's Republic \\ of China; ${ }^{2}$ Department of PET/CT Center, Harbin Medical University Cancer Hospital, Harbin, Heilongjiang Province, I5008I, People's Republic of China; \\ ${ }^{3}$ Department of Medical Oncology, Harbin Medical University Cancer Hospital, Harbin, Heilongjiang Province, I5008I, People's Republic of China; \\ ${ }^{4}$ Department of Pathology, Harbin Medical University Cancer Hospital, Harbin, Heilongjiang Province, I5008I, People's Republic of China; ${ }^{5}$ Department of \\ Medical Oncology, The Fourth Affiliated Hospital Zhejiang University School of Medicine, Yiwu, Zhejiang Province, 322000, People's Republic of China
}

Correspondence: Qijia Xuan, Department of Medical Oncology, The Fourth Affiliated Hospital, Zhejiang University School of Medicine, Yiwu, Zhejiang Province, 322000, People's Republic of China, Tel +86-579-582303, Email xuanqijia@zju.edu.cn

Background: Several reports have suggested that glucose transporter 3 (GLUT-3) promotes tumor metastasis. The aim of this study was to examine the relationship between the expression level of GLUT-3 and the prognosis of patients with diffuse large B cell lymphoma (DLBCL).

Methods: The GLUT-3 expression levels in 91 DLBCL patients were evaluated by immunohistochemistry. The relationships between GLUT-3 expression level and clinicopathological characteristics and progression-free survival (PFS) of DLBCL patients were analyzed. The use of validation cohorts confirmed the predictive value of GLUT-3 expression. The correlation between GLUT-3 and immune cell infiltration was investigated using the Cell-type Identification By Estimating Relative Subsets Of RNA Transcripts system and the analysis of the infiltrating score was obtained by single sample Gene Set Enrichment Analysis.

Results: Expression of GLUT-3, which is highly expressed in DLBCL patients, was significantly associated with elevated serum LDH level, recurrence and Ki-67 status. Kaplan-Meier analysis showed that high GLUT-3 expression levels in DLBCL were related to poor PFS. Univariate and multivariate analyses results showed that low GLUT-3 expression level was significantly but independently associated with favorable PFS in DLBCL patients. GLUT-3 expression was also correlated with immune cell infiltration and the analysis of the infiltrating score.

Conclusion: Our results indicate that GLUT-3 may act as a potential independent prognostic factor in DLBCL patients. The difference of the immune microenvironment in DLBCL patients may be predicted by the expression level of GLUT-3.

Keywords: glucose transporter 3, diffuse large B cell lymphoma, prognosis, biomarker

\section{Introduction}

Diffuse large B cell lymphoma (DLBCL), the most frequent subtype of lymphoid malignancy, remains a significant clinical challenge, as approximately $30 \%$ of patients are not cured after standard immunochemotherapy. ${ }^{1}$ The therapyresistant tumors exhibit both increased invasiveness and increased proliferation. ${ }^{1-3}$ Therefore, identifying novel therapeutic targets of DLBCL is urgently required to improve the prognoses of patients with DLBCL.

Metabolic remodeling during tumor progression is a hallmark of tumors. Most tumors exhibit aerobic glycolysis, also known as the Warburg effect. ${ }^{4-7}$ Reprogramming of energy metabolism provides energy for tumor growth, invasion, metastasis and resistance to therapeutics. ${ }^{89}$ The enhanced glycolysis of tumor cells affects the tumor immune microenvironment and help tumors survive by promoting immunosuppression. ${ }^{10,11}$ Therefore, interventions targeting energy metabolism in tumor cells may be an effective strategy to reduce or reverse therapeutic resistance.

The glucose transporter (GLUT) family is a family of key gating proteins that are responsible for the transport of glucose in and out of cells. GLUTs are responsible for the energy supply of cells and the regulation of glucose levels. In humans, the GLUT family has 14 members, and they function to transport glucose to different tissues. ${ }^{12}$ GLUT-1 is widely expressed in normal tissues and is overexpressed in many tumors. ${ }^{13}$ GLUT- 3 is expressed in neuronal cells and 
expressed at low levels in other organs under normal conditions. ${ }^{14}$ Recent studies have showed that GLUT-3 is overexpressed in many solid tumors, such as non-small cell lung carcinoma, hepatocellular carcinoma, and bladder cancer, and overexpressed GLUT-3 in tumor cells may be related to the rapid proliferation of cancer cells in hypoxic conditions. ${ }^{15-17}$ Since the rate of ATP production by glycolysis under anaerobic conditions is significantly lower than that of aerobic metabolism, tumor cells need high levels of GLUTs to meet the increased demand for glucose. GLUT-3 is overexpressed in many tumor cells, suggesting that it may act as a potential marker of tumor cells. However, the expression and prognostic value of GLUT-3 in DLBCL are largely unknown.

In this study, we evaluated GLUT-3 expression level in DLBCL to assess the relationship of GLUT-3 expression with the clinicopathological characteristics and clinical prognosis of DLBCL patients. We also explored the relationship between GLUT-3 expression in DLBCL and immune cells in the tumor microenvironment.

\section{Materials and Methods}

\section{Patients}

The clinicopathological data of 91 patients who were diagnosed with DLBCL between January 2008 and December 2012 at Harbin Medical University Cancer Hospital were retrospectively reviewed for this study. The inclusion criteria were as follows: i) CD20 positive DLBCL; ${ }^{18}$ ii) patients had no kidney, liver, heart or other serious somatic diseases; iii) no evidence of primary cancer in other organs; and iv) complete clinical data and follow-up information was available. These patients received standard R-CHOP (rituximab, cyclophosphamide, vincristine, doxorubicin, and prednisone) treatment for 6-8 cycles. During follow-ups, appropriate diagnostic examinations were used to confirm disease relapse in patients. Progression-free survival (PFS) was calculated from the date of diagnosis to the date of disease recurrence, death or the end of follow-up.

Information on baseline characteristics was collected, including sex, age, presence of B symptoms, Ann Arbor stage, Eastern Cooperative Oncology Group performance status (ECOG PS), laboratory data (LDH level), number of extranodal sites, bone marrow findings, bulky disease and Ki-67 status. International Prognostic Index (IPI) included age, ECOG, serum LDH, Ann Arbor stage and extranodal sites. ${ }^{19}$ The patients were divided into germinal center B cell (GCB) and non-GCB subtypes according to the Hans criteria. This study was approved by the Institutional Review Board of Harbin Medical University Cancer Hospital.

\section{Immunohistochemistry}

Immunohistochemistry was performed as previously reported. ${ }^{20}$ Briefly, the tissue was fixed with $37 \%$ formaldehyde solution and then paraffin-embedded to prepare tissue sections, which were approximately $4 \mu \mathrm{m}$ thick. Staining was performed using the anti-GLUT-3 polyclonal antibody (1:50 dilution, ab95256, Abcam). GLUT-3 expression was confirmed by the positive staining of the cell membrane. Tumor cells that showed a strong membrane signal for GLUT-3 were counted using a microscope in ten low magnification fields, and the result was calculated as a percentage of the total cell numbers. ${ }^{16}$ Samples with more than $10 \%$ positively stained cells were classified as high GLUT-3 expression.

\section{Gene Expression Omnibus (GEO) Database Analysis}

GEO (http://www.ncbi.nlm.nih.gov/geo/) is an international public repository containing high-throughput microarray and next-generation sequencing functional genomic data sets. ${ }^{21}$ All genes from two independent DLBCL GEO cohorts [GSE117556, 928 samples; GSE31312, 498 samples] were analyzed.

\section{Estimation of Immune Infiltration}

We used gene expression data downloaded from the GEO database to evaluate the difference of immune infiltration level between the high and low GLUT-3 expression groups. GSE31312 is a human DLBCL expression profile that was sequenced using the GPL570 platform (Affymetrix Human Genome U133 Plus 2.0 Array); it contains 498 samples of DLBCL. GSE117556 is another human DLBCL expression profile containing 928 samples sequenced by the GPL14951 
platform (Illumina HumanHT-12 WG-DASL V4.0 R2 expression BeadChip). For the analysis of tumor immune infiltration, we used two different methods for corroboration: Cell-type Identification By Estimating Relative Subsets Of RNA Transcripts (CIBERSORT) and Single Sample Gene Set Enrichment Analysis (ssGSEA).

\section{CIBERSORT Database Analysis}

CIBERSORT (http://cibersort.stanford.edu) allows for the estimation of the abundance of different types of cells by analyzing a large amount of transcription data. ${ }^{22}$ We used CIBERSORT to gain deeper insights into the tumor microenvironment of DLBCL. The abundance ratio matrix of 22 immune cell types were obtained from GSE117556 and GSE31312 using R software to obtain patients with different levels of GLUT-3 expression. P $<0.05$ was considered to indicate statistical significance.

\section{ssGSEA Statistical Analyses}

The ssGSEA algorithm, which is based on 29 immune gene sets (the infiltrating score of 16 immune cells and the activity of 13 immune-related pathways), was applied to comprehensively quantify the relative abundance of immune cell types, pathways, functions, and checkpoints in each patient. The differences between the GLUT-3 low-expression group and high-expression group patients were analyzed using the GSVA R software package.

Data and statistical analyses were performed using the SPSS platform, version 20.0 (IBM, USA). The relationship of GLUT-3 expression level with patient and tumor characteristics were analyzed by the Fisher's exact test or the Chisquare test. The impact of GLUT-3 expression on PFS was analyzed using the Kaplan-Meier method. The two-tailed Log rank test was used to analyze the survival data between different groups. The Cox proportional hazard model was used in univariate and multivariate analysis to assess the variables in the prognostic factors section to assess their impact on PFS. $\mathrm{P}<0.05$ was considered to indicate statistical significance.

\section{Results}

\section{Clinicopathological Characteristics and Expression of GLUT-3 in Patients with DLBCL} Clinicopathological characteristics were presented in Table 1. There were 56 (61.5\%) males and 35 (38.5\%) females, with a mean age of 55 (range from 13 to 86 ) years. 13 (14.3\%) patients had positive B symptoms. The majority of the patients (60 cases, 67\%) had localized disease (Ann Arbor stage I-II). Based on the ECOG, 84 (92.3\%) patients were in group 01. At diagnosis, the median LDH was 246U/L (range from 98-2353.6U/L).

We next performed an immunohistochemical study to investigate GLUT-3 expression on DLBCL tissues, and representative IHC images are presented in Figure 1. GLUT-3 expression was detected in DLBCL tissues. We categorized cases based on high and low GLUT-3 expression as described in Methods and examined associations with clinicopathological characteristics. High GLUT-3 expression in DLBCL was significantly and positively associated with elevated LDH level $(\mathrm{P}=0.047)$, recurrence $(\mathrm{P}=0.030)$ and $\mathrm{Ki}-67$ status $(\mathrm{P}=0.009)$.

\section{Survival of Patients with DLBCL with High and Low GLUT-3 Expression}

During the follow-up, 43 patients (47.3\%) showed no evidence of any disease progression, while 48 patients $(52.7 \%)$ showed progressive disease or death. We evaluated GLUT-3 expression level and PFS using Kaplan-Meier survival analysis. The result showed that patients infused with a low expression of GLUT-3 showed a longer PFS compared with that of patients infused with a high expression of GLUT-3 $(\mathrm{P}=0.035)$ (Figure 2).

\section{Predictors for PFS in DLBCL Patients}

We evaluated the relationship of clinicopathologic factors and PFS using univariable analysis. The results showed that GLUT-3 expression (Hazard Ratio [HR]: 2.074, 95\% Confidence Interval [CI] $=1.033-4.167, \mathrm{P}=0.040$ ) and IPI (HR:1.845, 95\% CI=1.108-3.073, $\mathrm{P}=0.019$ ) significantly indicated the prognosis of patients by predicting PFS (Table 2). Multivariate analysis identified GLUT-3 expression (HR: $2.255,95 \% \mathrm{CI}=1.083-4.693, \mathrm{P}=0.030$ ) as an 
Table I Characteristics of Diffuse Large B-Cell Lymphoma According to GLUT-3 Expression

\begin{tabular}{|c|c|c|c|c|}
\hline \multirow[t]{2}{*}{ Characteristic } & \multirow[t]{2}{*}{$N(\%) n=91$} & \multicolumn{2}{|c|}{ GLUT3 } & \multirow[t]{2}{*}{ P-value } \\
\hline & & High ( $\geq 10 \%)$ & Low (<10\%) & \\
\hline \multicolumn{4}{|l|}{ Sex } & \multirow[t]{3}{*}{0.078} \\
\hline Male & $56(61.5)$ & $35(55.6)$ & $21(75)$ & \\
\hline Female & $35(38.5)$ & $28(44.4)$ & $7(25)$ & \\
\hline \multicolumn{5}{|l|}{ Age } \\
\hline$\leq 60$ years & $53(58.2)$ & $37(58.7)$ & $16(57.1)$ & \multirow[t]{2}{*}{0.887} \\
\hline$>60$ years & $38(4 I .8)$ & $26(41.3)$ & $12(42.9)$ & \\
\hline \multicolumn{4}{|c|}{ Presence of B symptoms } & \multirow[t]{3}{*}{0.052} \\
\hline No & 78 (85.7) & $57(90.5)$ & $21(75)$ & \\
\hline Yes & $13(14.3)$ & $6(9.5)$ & $7(25)$ & \\
\hline \multicolumn{4}{|l|}{ Ann Arbor stage } & \multirow[t]{3}{*}{0.911} \\
\hline $\mathrm{I} / \mathrm{II}$ & $61(67)$ & $42(66.7)$ & $19(67.9)$ & \\
\hline III/IV & $30(33)$ & $21(33.3)$ & $9(32.1)$ & \\
\hline \multicolumn{4}{|c|}{ Performance status } & \multirow[t]{3}{*}{0.896} \\
\hline ECOG 0-I & $84(92.3)$ & $58(92.1)$ & $26(92.9)$ & \\
\hline$E C O G \geq 2$ & $7(7.7)$ & $5(7.9)$ & $2(7.1)$ & \\
\hline \multicolumn{4}{|l|}{ LDH level } & \multirow[t]{3}{*}{0.047} \\
\hline$<246$ U/L & $28(3 \mathrm{I})$ & $12(23)$ & $16(42)$ & \\
\hline$\geq 246 \mathrm{U} / \mathrm{L}$ & $63(69)$ & $4 \mid(77)$ & $22(58)$ & \\
\hline \multicolumn{4}{|c|}{ Number of extranodal sites } & \multirow[t]{4}{*}{0.685} \\
\hline $0-1$ & $85(93.4)$ & $58(92.1)$ & $27(96.4)$ & \\
\hline $2-3$ & $5(5.5)$ & $4(6.3)$ & I (3.6) & \\
\hline $4-5$ & $I(I .1)$ & I (I.6) & $0(0)$ & \\
\hline \multicolumn{4}{|c|}{ Bone marrow involvement } & \multirow[t]{3}{*}{0.325} \\
\hline Absence & $84(92.3)$ & $57(90.5)$ & $27(96.4)$ & \\
\hline Presence & 7 (7.7) & $6(9.5)$ & I (3.6) & \\
\hline \multicolumn{4}{|l|}{ Bulky disease } & \multirow[t]{3}{*}{0.431} \\
\hline No & $70(76.9)$ & $47(74.6)$ & $23(82.1)$ & \\
\hline Yes & $21(23.1)$ & $16(25.4)$ & $5(17.9)$ & \\
\hline \multicolumn{4}{|l|}{ Subtype } & \multirow[t]{3}{*}{0.970} \\
\hline GCB & 29 (31.9) & $20(31.7)$ & $9(32.1)$ & \\
\hline Non-GCB & $62(68.1)$ & $43(67.9)$ & 19 (67.9) & \\
\hline
\end{tabular}

(Continued) 
Table I (Continued).

\begin{tabular}{|c|c|c|c|c|}
\hline \multirow[t]{2}{*}{ Characteristic } & \multirow[t]{2}{*}{$N(\%) n=91$} & \multicolumn{2}{|c|}{ GLUT3 } & \multirow{2}{*}{$\begin{array}{c}\text { P-value } \\
0.526\end{array}$} \\
\hline & & High ( $\geq 10 \%)$ & Low $(<10 \%)$ & \\
\hline $0-1$ & $72(79.1)$ & $48(76.2)$ & $24(85.7)$ & \\
\hline $2-3$ & $18(19.8)$ & 14 (22.2) & $4(14.3)$ & \\
\hline $4-5$ & I (I.I) & I (I.6) & $0(0)$ & \\
\hline \multicolumn{4}{|l|}{ Recurrence } & 0.030 \\
\hline Yes & $48(52.7)$ & $38(60.3)$ & $10(35.7)$ & \\
\hline No & $43(47.3)$ & $25(39.7)$ & $18(64.3)$ & \\
\hline \multicolumn{4}{|l|}{ Ki-67 } & 0.009 \\
\hline$<80 \%$ & $43(47.3)$ & $24(38.1)$ & $19(67.9)$ & \\
\hline$\geq 80 \%$ & $48(52.7)$ & 39 (61.9) & $9(32.1)$ & \\
\hline
\end{tabular}

Abbreviations: GLUT-3, glucose transporter 3; GCB, germinal center B cell; IPI, International Prognostic Index.

independent prognostic factor (Table 2). In univariate or multivariate analysis, there was no significant correlation with PFS and Ki-67, sex, age, Ann Arbor stage and number of extranodal sites.

\section{GLUT-3 is Correlated with Immune Cell Infiltration in DLBCL}

We further compared the infiltration levels of immune cells between low and high GLUT-3 expression groups using CIBERSORT and ssGSEA. The infiltration levels of CD4 memory activated T cells, gamma delta $(\gamma \delta) \mathrm{T}$ cells and neutrophils in the group with high GLUT-3 expression were higher compared with those observed in the group with low GLUT-3 expression after statistical analysis of CIBERSORT results (Figure 3A-D). Statistical analysis of ssGSEA results also showed that the infiltration level of macrophages, neutrophils, $\mathrm{T}$ helper cells, tumor infiltrating lymphocytes (TILs), and regulatory T cells (Tregs) were significantly higher in the GLUT-3 high-expression group, while the GLUT-3 high-expression group was significantly enriched in most immune functions (except APC co-stimulation and HLA) (Figure 3B, C, E and F).

\section{Discussion}

The two most important factors in treatment strategies selecting and DLBCL prognosis predicting are clinical features and molecular pathology. ${ }^{23}$ However, more acceptable markers should be explored according to standard criteria. Our

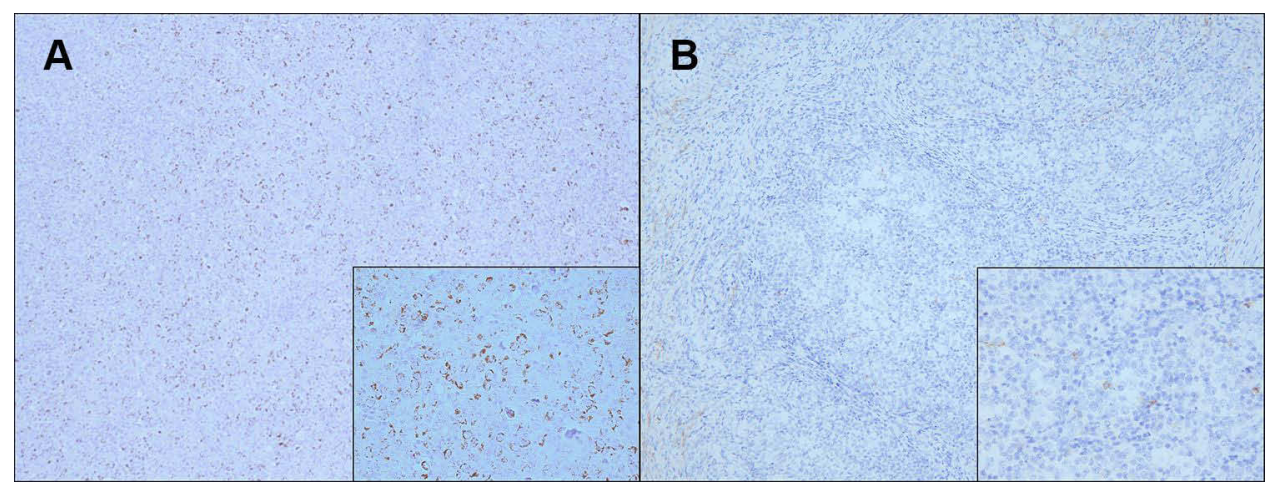

Figure I Representative immunohistochemistry images of GLUT-3 expression in diffuse large B-cell lymphoma tissues $(\times 100, \times 400)$. $((\mathbf{A})$ Diffuse large B-cell lymphoma tissues with high GLUT-3 expression); (B) Diffuse large B-cell lymphoma tissues with low GLUT-3 expression.

Abbreviation: GLUT-3, glucose transporter 3. 


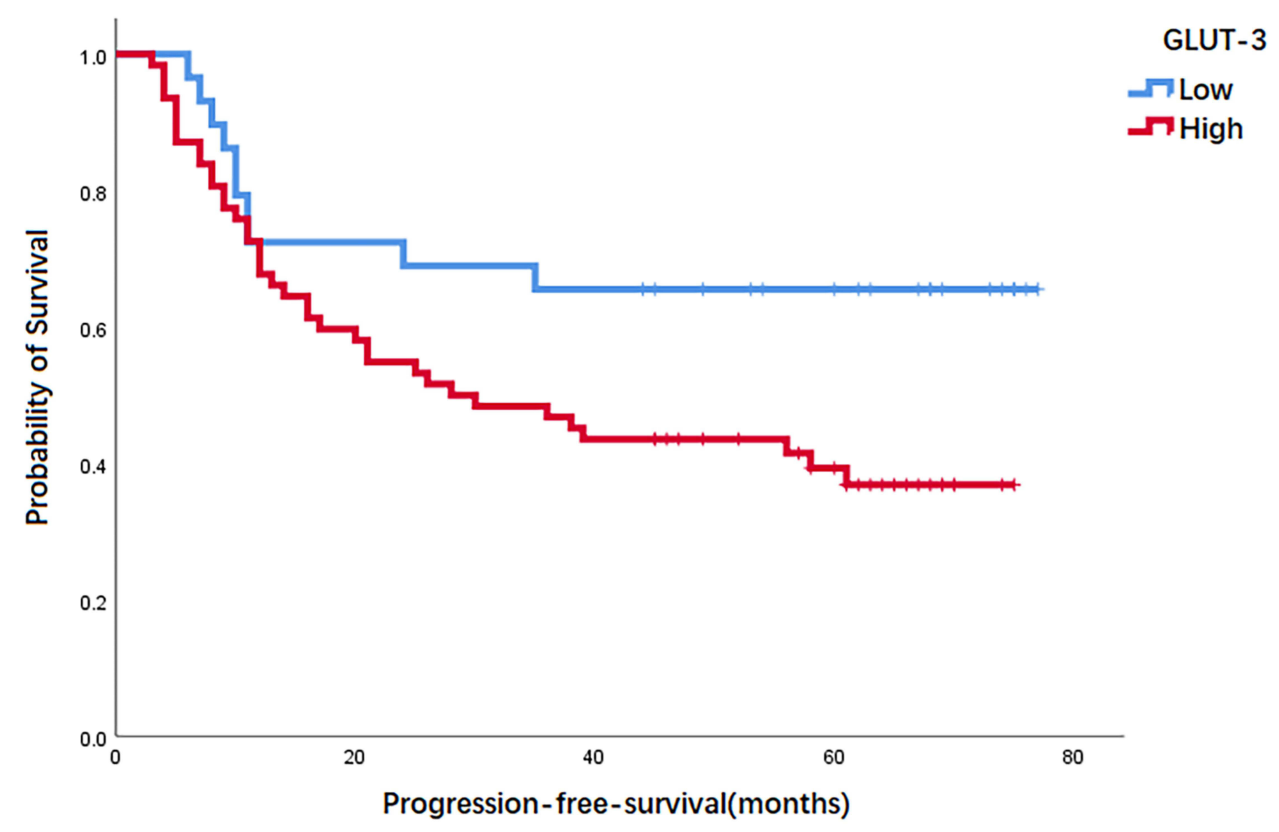

Figure 2 Kaplan-meier curves for PFS according to expression of GLUT-3 in patients with diffuse large B-cell lymphoma. Abbreviations: GLUT-3, glucose transporter 3; PFS, progression-free survival.

study showed that the increased GLUT-3 expression level was associated with the decreased PFS in DLBCL patients treated with standard R-CHOP chemotherapy. GLUT-3 expression level was also associated with elevated serum LDH level, recurrence and Ki-67 status. Taken together, these results demonstrated that GLUT-3 overexpression may be a biomarker to predict the PFS of DLBCL patients.

GLUTs are a family of transporters that mediate the transport of biological macromolecules such as monosaccharides, polyols, and other small carbon compounds across eukaryotic cell membranes. ${ }^{24}$ GLUT-3 belongs to the type I GLUT family; GLUT-3 was first discovered in fetal skeletal muscle cells and later found to be dominant in nerves and brain. Although GLUT-3 expression is tissue-specific, high expression of GLUT-3 has been observed in various tumors including gastric cancer, colon cancer, and brain tumors. ${ }^{25,26}$ Previous studies have reported that GLUT-3 is upregulated in tumor cells inhibited by bevacizumab and has been implicated in antiangiogenic resistance therapy and tumor stem cell phenotypes. ${ }^{27,28}$ GLUT-3 has highly active glucose transporter capacity. Compared with other class I GLUT family members, GLUT-3 has a higher affinity and higher transport capacity for glucose, and it has a five-fold higher affinity than the widely expressed GLUT-1. ${ }^{29}$

In recent years, GLUT-3 has been reported as a potential target for antitumor drug therapy. ${ }^{30-33}$ To our knowledge, the present study was the first to analyze GLUT-3 expression and its association with the prognosis of DLBCL patients.

Table 2 Univariate and Multivariate Analyses of Clinicopathologic Characteristics for DFS in Diffuse Large B-Cell Lymphoma

\begin{tabular}{|l|c|c|c|c|}
\hline \multirow{2}{*}{ Variable } & \multicolumn{2}{|c|}{ Univariate Analysis } & \multicolumn{2}{c|}{ Multivariate Analysis } \\
\cline { 2 - 5 } & HR (95\% Cl) & P-value & HR(95\% Cl) & P-value \\
\hline GLUT-3 & $2.074(1.033-4.167)$ & 0.040 & $2.255(I .083-4.693)$ & 0.030 \\
IPI & $1.845(1.108-3.073)$ & 0.019 & $1.208(0.463-3.15 I)$ & 0.700 \\
Ki-67 & $1.17 I(0.663-2.067)$ & 0.587 & $0.922(0.499-1.705)$ & 0.795 \\
Sex & $1.169(0.647-2.112)$ & 0.605 & $1.377(0.740-2.562)$ & 0.312 \\
Age & $1.418(0.804-2.499)$ & 0.227 & $1.811(0.918-3.574)$ & 0.087 \\
Ann Arbor stage & $1.674(0.942-2.977)$ & 0.079 & $1.691(0.744-3.84 I)$ & 0.210 \\
Number of extranodal sites & $1.46 I(0.719-2.970)$ & 0.294 & $1.046(0.366-2.988)$ & 0.934 \\
\hline
\end{tabular}

Abbreviations: GLUT-3, glucose transporter 3; IPI, international prognostic index. 
A

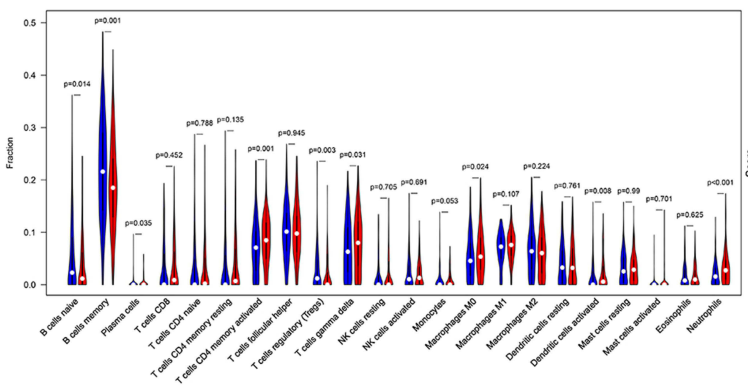

B

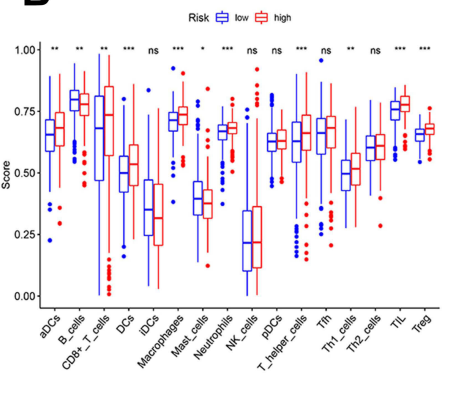

C

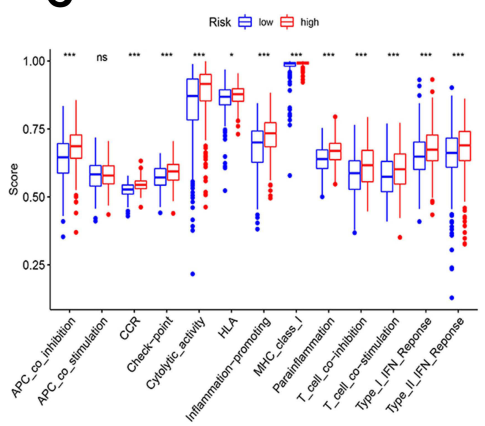

$\mathbf{F}$
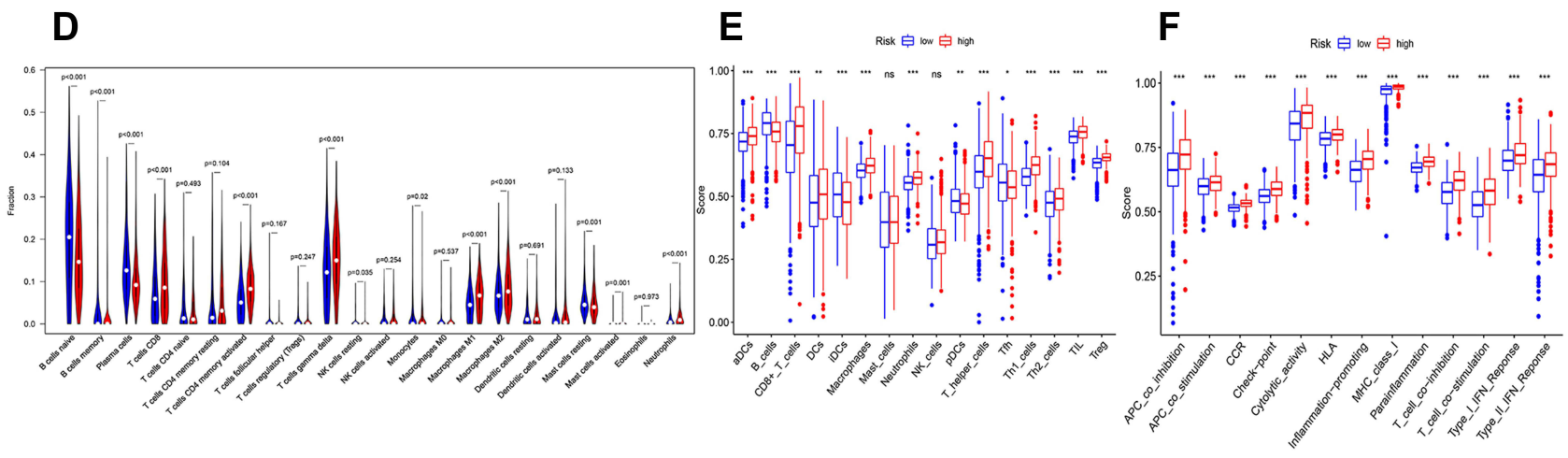

Figure 3 Boxplots and violin plots showing the infiltration levels of immune cell types in low-and high GLUT-3 expression groups. ((A) Infiltration levels of immune cell types by CIBERSORT in GSE3।3I2; (B) Infiltration levels of immune cell types by ssGSEA in GSE3 I3।2; (C) Immunecell functions by ssGSEA in GSE3 I I 2; (D) Infiltration levels of immune cell types by CIBERSORT in GSEI I7556; (E) Infiltration levels of immune cell types by ssGSEA in GSEI I 7556; (F) Immunecell functions by ssGSEA in GSEI I 7556). $*_{p}<0.05, * * p<0.01$, **** $<0.001$.

Abbreviations: GLUT-3, glucose transporter 3; ns, no statistically significant.

Malignant cells grow faster and require more oxygen and glucose than normal cells. Although mitochondrial oxidative phosphorylation is a more efficient process of ATP anabolism than glycolysis, tumor cells still rely on glycolysis as the primary metabolic mode, even in the presence of sufficient oxygen; this phenomenon is called the Warburg effect. ${ }^{34-36}$ In our study, we found that the expression of GLUT-3 was associated with Ki-67 status and the level of LDH. Although glycolysis produces less ATP, a large number of intermediate metabolites can be used to build macromolecular structures, including lipids, proteins, RNA, and NADP. ${ }^{37-39}$ As the tumor grows, cells may be exposed to hypoxia, leading to the production of hypoxia-inducible factor 1 (HIF-1) transcription factors, which increase the transcription of glucose transporters ${ }^{40,41}$ The decreased efficiency of ATP production in tumor cells and the high energy requirements of tumor cells can stimulate the increase of glucose uptake in malignant tumor cells. In addition, GLUT-3 overexpression is involved in increasing glucose transport to tumor cells to meet their high metabolism and rapid growth needs.

GLUT-3 is involved in multiple pathways, including cAMP, NF-kB, and p53 signaling pathways. ${ }^{42-45}$ In a recent study by Zhuang et al, GLUT-3 siRNA combined with vincristine (VCR) was shown to inhibit cell proliferation and promote cell apoptosis. The authors showed that GLUT-3 depletion downregulated BCR-ABL fusion gene expression, leading to increased caspase-3 and PTEN expression, which decreased intracellular VCR efflux, and thus enhanced the sensitivity of leukemia cells to VCR. ${ }^{46}$

Recent breakthroughs in immunotherapy have established novel strategies for the effective treatment of tumors. The discovery of reliable and powerful biomarkers, in addition to the pathological and clinical indexes already in use, is required to improve the individualized treatment of patients. Our results also demonstrated that there were more CD8 T cells, CD4 memory-activated T cells, $\gamma \delta$ T cells and macrophages in the high GLUT-3 expression group. GLUT-3 expression level was correlated with these immune markers. ssGSEA analysis also found the similar results: in the enrichment analysis of 13 immune functions and pathways, there was significant difference between the high- and lowexpression groups of GLUT-3 ( $\mathrm{p}<0.001$ ), indicating an effect of GLUT-3 on immune function and pathways. In the 
analysis of immune infiltration in high- and low-expression groups, both CIBERSORT and ssGSEA results suggested that GLUT-3 expression is related to the expression of different immune cells (CD4 memory-activated T cells, $\gamma \delta$ T cells, neutrophils, Tregs) in the tumor microenvironment. This genetic signature can predict the prognosis of DLBCL patients and provide some reference for immunotherapy.

Although the normal function of neutrophils is to kill microorganisms, increasing evidence has proven the key roles of tumor-associated neutrophils in tumor immune surveillance, metastasis and proliferation. In addition, some studies have shown that tumor-associated neutrophils can promote the survival of DLBCL cells and that neutrophils are also associated with poor prognosis of DLBCL. ${ }^{47}$ Fleming et al found that $\gamma \delta \mathrm{T}$ cells promote tumor progression by inducing an immunosuppressive tumor microenvironment and angiogenesis by IL-17. In addition, $\gamma \delta \mathrm{T}$ cells also can inhibit rejection of the tumor by mimicry of Tregs and Th-2 cell-like activity, restrain antitumor adaptive $\mathrm{T}$ cell immunity via the programmed death-1/programmed death ligand-1 pathway and use senescence as a mechanism to control antitumor immune responses in the tumor microenvironment. ${ }^{48}$ Zhong et al demonstrated that high abundance of TFT (Tim-3 +Foxp3+Treg) cells in the tumor microenvironment is predictive of poor outcomes of DLBCL. TFT cells promote DLBCL development partly by secreting IL-10 in the tumor microenvironment. Anti-Tim-3 antibodies, which block IL10 secretion, may represent an effective therapeutic agent for DLBCL. ${ }^{49}$

Cham and Gajewski recently reported that activated $\mathrm{T}$ cells require higher energy for proliferation and cytokine production. This process relies on glycolysis and therefore produces lactic acid; the glycolysis rate is increased only by continuously removing lactic acid in T cells. Since lactate anions cannot pass through the plasma membrane and diffuse freely, cells need a specific transport system, the monocarboxylic acid transporter (MCT), to excrete lactic acid. MCTmediated lactic acid excretion depends on the concentration gradient of lactic acid inside and outside the cell. Accumulation of lactic acid results in the obstruction of lactic acid excretion in $\mathrm{T}$ cells, which inhibits the secretion of IL-2 and IFN- $\gamma$ by T cells. ${ }^{50}$ Tumor cells highly express GLUT-3, enhance glycolysis, and cause lactic acid accumulation in the surrounding environment, which affects the antitumor effect of $\mathrm{T}$ cells. However, the mechanism of GLUT-3 overexpression in DLBCL and its relationship with the microenvironment is not clear. In the future, we will further study its potential mechanism and focus on evaluating the feasibility of GLUT-3 as a potential therapeutic target for DLBCL.

This study has several limitations. First, due to the retrospective nature of the study, there are inherent biases. Second, the study involved a relatively small number of patients, and the results should be confirmed in a larger cohort of patients. Third, the molecular mechanism of GLUT-3 overexpression in DLBCL tissue immune microenvironment is still unclear and needs further research.

\section{Conclusions}

This study demonstrated a correlation between GLUT-3 expression levels and clinical outcomes in DLBCL patients. The increased expression of GLUT-3 in DLBCL patients is associated with poor prognosis, which suggested that GLUT-3 may act as a potential prognostic factor for DLBCL. GLUT-3 expression in DLBCL may be related to the immunosuppressive microenvironment in tumors. These findings laid a foundation for GLUT-3 as a potential therapeutic target for DLBCL, and it may lead to the development of new therapeutic strategies.

\section{Abbreviations}

DLBCL, diffuse large B cell lymphoma; GLUT-3, glucose transporter 3; CIBERSORT, Cell-type Identification By Estimating Relative Subsets Of RNA Transcripts; ssGSEA, single sample Gene Set Enrichment Analysis; GLUT, glucose transporter; PFS, progression-free survival; IPI, International Prognostic Index; T $\gamma \delta$ cells, T gamma delta cells; TIL, tumor infiltrating lymphocytes; Tregs, regulatory T cells; R-CHOP, rituximab, cyclophosphamide, vincristine, doxorubicin, and prednisone; HIF-1, hypoxia-inducible factor 1; MCT, monocarboxylic acid transporter; VCR, vincristine; ECOG PS, Eastern Cooperative Oncology Group performance status; GCB, germinal center B cell. 


\section{Ethics Approval and Informed Consent}

This study was conducted in accordance with the Declaration of Helsinki. This study was approved by the Institutional Review Board of Harbin Medical University Cancer Hospital. All enrolled patients have signed informed consent.

\section{Acknowledgments}

We thank Gabrielle White Wolf, PhD, from Liwen Bianji (Edanz) (www.liwenbianji.cn/), for editing the English text of a draft of this manuscript.

\section{Author Contributions}

All authors made a significant contribution to the work reported, whether that is in the conception, study design, execution, acquisition of data, analysis and interpretation, or in all these areas; took part in drafting, revising or critically reviewing the article; gave final approval of the version to be published; have agreed on the journal to which the article has been submitted; and agree to be accountable for all aspects of the work.

\section{Funding}

This study was supported by the National Natural Science Foundation of China (Grant No.81800193).

\section{Disclosure}

The authors declare that they have no competing interests.

\section{References}

1. Kallam A, Adusumalli J, Armitage JO. Surveillance in patients with diffuse large B cell lymphoma. Mayo Clin Proc. 2020;95(1):157-163. doi:10.1016/j.mayocp.2019.05.011

2. Frontzek F, Lenz G. Novel insights into the pathogenesis of molecular subtypes of diffuse large B-cell lymphoma and their clinical implications. Expert Rev Clin Pharmacol. 2019;12(11):1059-1067. doi:10.1080/17512433.2019.1683447

3. Sukswai N, Lyapichev K, Khoury JD, Medeiros LJ. Diffuse large B-cell lymphoma variants: an update. Pathology. 2020;52(1):53-67. doi:10.1016/ j.pathol.2019.08.013

4. Sewastianik T, Szydlowski M, Jablonska E, et al. FOXO1 is a TXN- and p300-dependent sensor and effector of oxidative stress in diffuse large B-cell lymphomas characterized by increased oxidative metabolism. Oncogene. 2016;35(46):5989-6000. doi:10.1038/onc.2016.126

5. Buppajarntham S, Junpaparp P, Kue APP. Warburg effect associated with transformed lymphoplasmacytic lymphoma to diffuse large B-cell lymphoma. Am J Emerg Med. 2013;31(6):999-e5. doi:10.1016/j.ajem.2013.01.038

6. Caro P, Kishan AU, Norberg E, et al. Metabolic signatures uncover distinct targets in molecular subsets of diffuse large B cell lymphoma. Cancer Cell. 2012;22(4):547-560. doi:10.1016/j.ccr.2012.08.014

7. Chiche J, Pommier S, Beneteau M, et al. GAPDH enhances the aggressiveness and the vascularization of non-Hodgkin's B lymphomas via NF$\kappa B-d e p e n d e n t$ induction of HIF-1 $\alpha$. Leukemia. 2015;29(5):1163-1176. doi:10.1038/leu.2014.324

8. Lyssiotis CA, Kimmelman AC. Metabolic interactions in the tumor microenvironment. Trends Cell Biol. 2017;27(11):863-875. doi:10.1016/j. tcb.2017.06.003

9. Pavlova NN, Thompson CB. The emerging hallmarks of cancer metabolism. Cell Metab. 2016;23(1):27-47. doi:10.1016/j.cmet.2015.12.006

10. Li W, Xu M, Li Y, et al. Comprehensive analysis of the association between tumor glycolysis and immune/inflammation function in breast cancer. J Transl Med. 2020;18(1):92. doi:10.1186/s12967-020-02267-2

11. Cascone T, McKenzie JA, Mbofung RM, et al. Increased tumor glycolysis characterizes immune resistance to adoptive T cell therapy. Cell Metab. 2018;27(5):977-987.e974. doi:10.1016/j.cmet.2018.02.024

12. Lizak B, Szarka A, Kim Y, et al. Glucose transport and transporters in the endomembranes. Int J Mol Sci. 2019;20(23):5898. doi:10.3390/ ijms20235898

13. Koch H, Weber YG. The glucose transporter type 1 (Glut1) syndromes. Epilepsy Behav. 2019;91:90-93. doi:10.1016/j.yebeh.2018.06.010

14. Kuo CC, Ling HH, Chiang MC, et al. Metastatic colorectal cancer rewrites metabolic program through a Glut3-YAP-dependent signaling circuit. Theranostics. 2019;9(9):2526-2540. doi:10.7150/thno.32915

15. Massari F, Ciccarese C, Santoni M, et al. Metabolic phenotype of bladder cancer. Cancer Treat Rev. 2016;45:46-57. doi:10.1016/j.ctrv.2016.03.005

16. Gao H, Hao Y, Zhou X, et al. Prognostic value of glucose transporter 3 expression in hepatocellular carcinoma. Oncol Lett. 2020;19(1):691-699. doi:10.3892/ol.2019.11191

17. Masin M, Vazquez J, Rossi S, et al. GLUT3 is induced during epithelial-mesenchymal transition and promotes tumor cell proliferation in non-small cell lung cancer. Cancer Metab. 2014;2:11. doi:10.1186/2049-3002-2-11

18. Friedberg JW. New strategies in diffuse large B-cell lymphoma: translating findings from gene expression analyses into clinical practice. Clin Cancer Res. 2011;17(19):6112-6117. doi:10.1158/1078-0432.CCR-11-1073

19. International Non-Hodgkin's Lymphoma Prognostic Factors Project. A predictive model for aggressive non-Hodgkin's lymphoma. $N$ Engl $J$ Med. 1993;329(14):987-994. doi:10.1056/NEJM199309303291402 
20. Wang J, Gao K, Lei W, et al. Lymphocyte-to-monocyte ratio is associated with prognosis of diffuse large B-cell lymphoma: correlation with CD163 positive M2 type tumor-associated macrophages, not PD-1 positive tumor-infiltrating lymphocytes. Oncotarget. 2017;8(3):5414-5425. doi:10.18632/oncotarget.14289

21. Barrett T, Wilhite SE, Ledoux P, et al. NCBI GEO: archive for functional genomics data sets-update. Nucleic Acids Res. 2013;41:D991-D995. doi:10.1093/nar/gks1193

22. Newman AM, Liu CL, Green MR, et al. Robust enumeration of cell subsets from tissue expression profiles. Nat Methods. 2015;12(5):453-457. doi:10.1038/nmeth.3337

23. King RL, Goodlad JR, Calaminici M, et al. Lymphomas arising in immune-privileged sites: insights into biology, diagnosis, and pathogenesis. Virchows Archiv. 2019;476:647-665.

24. de la Cruz-lopez KG, Castro-Munoz LJ, Reyes-Hernandez DO, Garcia-Carranca A, Manzo-Merino J. Lactate in the regulation of tumor microenvironment and therapeutic approaches. Front Oncol. 2019;9:1143. doi:10.3389/fonc.2019.01143

25. Reckzeh ES, Waldmann H. Small-molecule inhibition of glucose transporters GLUT-1-4. Chembiochem. 2019;21(1-2):45-52. doi:10.1002/ cbic. 201900544

26. Ceballos J, Schwalfenberg M, Karageorgis G, et al. Synthesis of indomorphan pseudo-natural product inhibitors of glucose transporters GLUT-1 and -3. Angewandte Chemie. 2019;58(47):17016-17025. doi:10.1002/anie.201909518

27. Kuang R, Jahangiri A, Mascharak S, et al. GLUT3 upregulation promotes metabolic reprogramming associated with antiangiogenic therapy resistance. JCI Insight. 2017;2(2):e88815. doi:10.1172/jci.insight.88815

28. Marin-Hernandez A, Gallardo-Perez JC, Ralph SJ, Rodriguez-Enriquez S, Moreno-Sanchez R. HIF-1alpha modulates energy metabolism in cancer cells by inducing over-expression of specific glycolytic isoforms. Mini Rev Med Chem. 2009;9(9):1084-1101. doi:10.2174/ 138955709788922610

29. Holman GD. Chemical biology probes of mammalian GLUT structure and function. Biochem J. 2018;475(22):3511-3534. doi:10.1042/ BCJ20170677

30. Ancey PB, Contat C, Meylan E. Glucose transporters in cancer - from tumor cells to the tumor microenvironment. FEBS J. 2018;285 (16):2926-2943. doi:10.1111/febs.14577

31. Gonzalez-Menendez P, Hevia D, Mayo JC, Sainz RM. The dark side of glucose transporters in prostate cancer: are they a new feature to characterize carcinomas? Int J Cancer. 2018;142(12):2414-2424. doi:10.1002/ijc.31165

32. Labak CM, Wang PY, Arora R, et al. Glucose transport: meeting the metabolic demands of cancer, and applications in glioblastoma treatment. Am $J$ Cancer Res. 2016;6(8):1599-1608.

33. Patching SG. Glucose transporters at the blood-brain barrier: function, regulation and gateways for drug delivery. Mol Neurobiol. 2017;54 (2):1046-1077. doi:10.1007/s12035-015-9672-6

34. Kobliakov VA. The mechanisms of regulation of aerobic glycolysis (Warburg Effect) by oncoproteins in carcinogenesis. Biochemistry. 2019;84 (10):1117-1128. doi:10.1134/S0006297919100018

35. Abbaszadeh Z, Cesmeli S, Biray Avci C. Crucial players in glycolysis: cancer progress. Gene. 2020;726:144158. doi:10.1016/j.gene.2019.144158

36. Noguera R, Burgos-Panadero R, Gamero-Sandemetrio E, de la Cruz-merino L, Alvaro Naranjo T. [An integral view of cancer (II). Fields of investigation and emerging biomarkers]. Revista Española de Patologia. 2019;52(4):222-233. Spanish. doi:10.1016/j.patol.2019.04.005

37. Yu T, Wang Y, Fan Y, et al. CircRNAs in cancer metabolism: a review. J Hematol Oncol. 2019;12(1):90. doi:10.1186/s13045-019-0776-8

38. Hossain F, Andreana PR. Developments in carbohydrate-based cancer therapeutics. Pharmaceuticals. 2019;12(2). doi:10.3390/ph12020084

39. Qureshi AS, Ali S. Review: Warburg effect and renal cancer caused by errs in fumarate hydratase encoding gene. Pak J Pharm Sci. 2019;32 (2):743-749.

40. Moriyama H, Moriyama M, Ozawa T, et al. Notch signaling enhances stemness by regulating metabolic pathways through modifying $\mathrm{p} 53$, NF- $\mathrm{kB}$, and HIF-1 $\alpha$. Stem Cells Dev. 2018;27(13):935-947. doi:10.1089/scd.2017.0260

41. Yan YE, Zhang J, Wang K, et al. Significant reduction of the GLUT3 level, but not GLUT1 level, was observed in the brain tissues of several scrapie experimental animals and scrapie-infected cell lines. Mol Neurobiol. 2014;49(2):991-1004. doi:10.1007/s12035-013-8574-8

42. Zha X, Hu Z, Ji S, et al. NFkappaB up-regulation of glucose transporter 3 is essential for hyperactive mammalian target of rapamycin-induced aerobic glycolysis and tumor growth. Cancer Lett. 2015;359(1):97-106. doi:10.1016/j.canlet.2015.01.001

43. Wang Y, Wu S, Huang C, Li Y, Zhao H, Kasim V. Yin Yang 1 promotes the Warburg effect and tumorigenesis via glucose transporter GLUT3. Cancer Sci. 2018;109(8):2423-2434. doi:10.1111/cas.13662

44. Brito AF, Abrantes AM, Ribeiro M, et al. Fluorine-18 fluorodeoxyglucose uptake in hepatocellular carcinoma: correlation with glucose transporters and p53 expression. J Clin Exp Hepatol. 2015;5(3):183-189. doi:10.1016/j.jceh.2015.05.003

45. Watanabe M, Abe N, Oshikiri Y, Stanbridge EJ, Kitagawa T. Selective growth inhibition by glycogen synthase kinase-3 inhibitors in tumorigenic HeLa hybrid cells is mediated through NF-KB-dependent GLUT3 expression. Oncogenesis. 2012;1:e21. doi:10.1038/oncsis.2012.21

46. Zhuang Y, Zhao J, Xu X, Bi L. Downregulation of GLUT3 promotes apoptosis and chemosensitivity of acute myeloid leukemia cells via EGFR signaling. Arch Iran Med. 2018;21(2):73-78.

47. Nie M, Yang L, Bi X, et al. Neutrophil extracellular traps induced by IL8 promote diffuse large B-cell lymphoma progression via the TLR9 signaling. Clin Cancer Res. 2019;25(6):1867-1879. doi:10.1158/1078-0432.CCR-18-1226

48. Fleming C, Morrissey S, Cai Y, Yan J. $\gamma \delta$ T cells: unexpected regulators of cancer development and progression. Trends Cancer. 2017;3 (8):561-570. doi:10.1016/j.trecan.2017.06.003

49. Zhong W, Liu X, Zhu Z, Li Q, Li K. High levels of Tim-3(+)Foxp3(+)Treg cells in the tumor microenvironment is a prognostic indicator of poor survival of diffuse large B cell lymphoma patients. Int Immunopharmacol. 2021;96:107662. doi:10.1016/j.intimp.2021.107662

50. Fischer K, Hoffmann P, Voelkl S, et al. Inhibitory effect of tumor cell-derived lactic acid on human T cells. Blood. 2007;109(9):3812-3819. doi:10.1182/blood-2006-07-035972 


\section{Publish your work in this journal}

OncoTargets and Therapy is an international, peer-reviewed, open access journal focusing on the pathological basis of all cancers, potential targets for therapy and treatment protocols employed to improve the management of cancer patients. The journal also focuses on the impact of management programs and new therapeutic agents and protocols on patient perspectives such as quality of life, adherence and satisfaction. The manuscript management system is completely online and includes a very quick and fair peer-review system, which is all easy to use. Visit http://www.dovepress.com/testimonials.php to read real quotes from published authors.

Submit your manuscript here: https://www.dovepress.com/oncotargets-and-therapy-journal 